\title{
ORAL CAVITY AND PERIODONT MICROBIOME STATUS THROUGH EMOTIONAL STRESS
}

\section{O.N. Risovannaya', S.V. Melekhov', V. L. Popkov', Z.V. Lalieva', D.A. Domenyuk ${ }^{3}$}

\author{
'Department of Dentistry Faculty of Advanced Studies and Retraining \\ Specialists, Kuban State Medical University, Ministry of Healthcare of \\ Russian Federation, M. Sedina str., 4, Krasnodar, Russia, 350063. \\ E-mail:stomatologia.fpk@gmail.com, tel:+78612680210 \\ 2 Department of Prosthetic Dentistry, Kuban State Medical University, \\ Ministry of Healthcare of Russian Federation, M. Sedina str., 4, Krasnodar, \\ Russia, 350063. E-mail: corpus@ksma.ru, tel: +7861 2680210 \\ ${ }^{3}$ Department of general practice dentistry and child dentistry, Stavropol \\ State Medical University, Ministry of Healthcare of Russian Federation, 310, \\ Mira Street, Stavropol, Russia 355017.E-mail:domenyukda@mail.ru, \\ tel: +79188701205
}

ABSTRACT - AIM. To study the effect of emotional stress on the microbial status of the gingival sulcus, going through eubiosis and dysbiosis, observed in law enforcement officers. MATERIALS AND METHODS. The study involved 67 law enforcement officers aged 30-49. The microbiological study focused on the gingival sulcus total microbial contamination and its colonization with certain types of microbes, employing aerobic and anaerobic cultivation methods. RESULTS. When in a state of relative rest, $100 \%$ of the persons with no gum issues were observed to have the eubiotic state of the gingival sulcus microbial status, while in $100 \%$ of persons with mild periodontitis the microbial status of the gingival sulcus was found to be in a state of dysbiosis. Conclusion. The stressor effect along with a microbial homeostasis imbalance in the dentogingival gap causes an increase in the microbial associations imbalance, which manifests itself as a decrease in the commensal microflora and an increase in the potentially pathogenic (opportunistic) one.

KEYW ORDS - microbial status, gingival sulcus, emotional stress, dysbiosis, eubiosis, aerobes, anaerobes.

\section{INTRODUCTION}

Oral cavity and periodontal microbiome is one of the most investigated microbial communities. This is due to its complex composition and its abundant interaction with the human body. The oral cavity is one of the most densely populated human ecosystems, which contains over 1,000 different types of eubacteria, archaea, fungi, protozoa and viruses. The oral microbiota status is directly related to a wide range of health issues, such as oral cavity diseases (caries, periodontal diseases), diabetes, cardiovascular diseases, cancer etc. Oral microbiome has been shown to have
Article history:

Received 31 January 2019

Received in revised form 6 March 2019

Accepted 10 March 2019

a comprehensive effect on the development of diseases - the decisive role belongs not to a particular microorganism, yet a combination of these [1-7].

In the oral cavity, typically several standard types of biological material are studied, which reflect a particular microbial community status - saliva, soft plaque, subgingival and supragingival dental calculus, as well as the contents of the gingival sulcus and of the periodontal pocket. All these biotopes, except the gingival sulcus and periodontal pocket biocenosis, are extremely unstable and depend significantly on the oral hygiene type and intensity. The unique feature of the gingival sulcus biotope is that quantitative and qualitative changes in these microecological system's microbial communities can lead to major dental issues - gingivitis, periodontitis, and caries. Structural and functional disorders of the gingival sulcus biofilm result in pathological changes that are not only of local, yet also of systemic importance [8-12].

Modern life environment is full of stress inducing factors, which make the body respond with a set of psychological, physiological and biochemical reactions that are indicative of psychological and emotional stress. In particular, a high level of emotional effects can be seen in law enforcement officers who are exposed to excessive loads under various social conditions. In case of emergencies, the emotional pressure experienced by a law enforcement officer goes up many times reaching the top peak of psycho-emotional stress $[13,14]$.

Most researchers studied the effect of emotional stress on the body's microbiome relying on the intestinal microbiota example. Stress factors have been proven to promote the intestinal microbiota imbalance and the growth of pathogenic microflora $[15,16]$. A limited number of works highlight the effect of emotional stress on the oral cavity microbial status. It is important to study the level of the effect that emotional factors have on the oral cavity areas that are responsible for the development of the most common dental diseases, such as periodontal tissues inflamma- 
tory diseases $[17,18]$. Studying changes in the gingival sulcus microbial status under various microecological situations in the oral cavity would allow a deeper analysis into the interaction between bacteria and the immune response under stress-response of the body [19].

To elucidate the effect of law enforcement officers' Aim of study emotional stress on the microbial status of the gingival sulcus, which is under eubiosis and dysbiosis.

\section{MATERIALS AND METHODS}

The study involved 67 law enforcement officers aged 30-49 years with no somatic diseases. Group 1 included 22 persons ( 11 males, 11 females) who had no clinical manifestation of periodontal or dental lesions. Group 2 included 45 persons ( 29 males, 16 females) diagnosed with chronic moderate periodontitis.

Law enforcement officer implies emotional stress - interaction with criminals, strict deadline for important tasks, night shifts. We used the C.D. Spielberger scale (adjusted by Yu.L. Khanin) to determine the level of personal and reactive anxiety. An indicator below 30 revealed low anxiety; $30-45$ points - moderate anxiety, while exceeding 45 points - showed high anxiety [20].

A microbiological study was carried for the total microbial contamination of the gingival sulcus and its colonization with certain microbial species; the methods used were those involving aerobic and anaerobic cultivation [21]. Samples of the gingival sulcus were taken in the morning on an empty stomach, with a sterile standard-size paper endodontic pin (\#30), $1 \mathrm{~cm}$ long, which, after soaking, was put in a sterile saline solution and washed thoroughly. Standard dilutions were plated on special, selective and differential diagnostic media - blood agar, yolk-salt agar, Sabuoraud's medium, Endo medium, sugar agar, followed with cultivation under aerobic and anaerobic conditions. For the inoculations obtained under aerobic cultivation conditions, the gingival fluid microbial content with aerobic and facultatively aerobic bacteria (further called aerobes) was identified. On the inoculations obtained under anaerobic cultivation conditions, microbial colonization of facultative and obligate anaerobes was determined (hereinafter referred to as anaerobes). Identification of the isolated pure cultures was carried out following morphological, tinctorial, cultural, and biochemical features. The results of the quantitative study were expressed in a decimal logarithm of colonyforming units per $1 \mathrm{ml}-\lg \mathrm{CFU} / \mathrm{ml}$. The frequency of identifying certain species of colonizing microflora was identified, too. The statistical analysis of results was performed using SPSS 17.0 and Microsoft Excel 2007 software. The total sample was analyzed with parametric methods after a preliminary check for the normal distribution with the Kolmogorov-Smirnov test. The differences between the studied parameters were evaluated with Student's t-test. The detection rates for individual microorganisms were compared with the $\varphi$ Fisher criterion [22].

\section{RESULTS AND DISCUSSION}

The first stage of the study involved identifying the gingival sulcus microbial condition (eubiosis or dysbiosis) in individuals with different dental status in a state of relative rest.

The microbial homeostasis of the gingival sulcus biofilm in individuals with periodontitis was different from that in persons with intact gums (Table 1). In the state of relative rest, the microbial load on the gingival sulcus biofilm in Group 2 exceeded the same index in Group 1. Our studies revealed that gingival sulcus colonization with aerobic and anaerobic microflora in people with periodontitis exceeded the same indicators in people with intact gums, 3.4 times $(\mathrm{p}<0.05)$ and 8.3 times $(\mathrm{p}<0.05)$, respectively.

The qualitative composition of the gingival sulcus microbial status in relative rest depended on the dental status, too. The frequency of the gingival sulcus colonization with S.viridans spp. in persons with periodontitis was $63.9 \%$ lower $(\mathrm{p}<0.05)$ than in those without gums issues (Tables 2 and 3). Also, the development of dental diseases came along with a 3.0 times decrease $(p<0.05)$ in the density of colonization of the gingival sulcus with S.viridans spp. (Table 2).

The gingival sulcus colonization with $\gamma$-hemolytic streptococci, which include S.mutans, in patients with periodontitis is 3.5 times $(\mathrm{p}<0.05)$ above that of persons without the pathology.

Neisseria spp. were $37.3 \%(\mathrm{p}<0.05)$ more often detected in people with periodontitis, while the colonization density with Neiserias was also higher $(28.2$ times $(\mathrm{p}<0.05))$ in that group, compared with those with no gums issues.

The density of epidermal staphylococcus colonization in patients with periodontitis was 3.2 times ( $\mathrm{p}$ $<0.05$ ) above that in healthy individuals. In Group 2, potentially pathogenic (opportunistic) microorganisms were more common (the percentage of increase is bracketed): Bacillus spp. $(20.4 \%(\mathrm{p}<0.05))$, S.aureus (21.1\% ( $\mathrm{p}<0.05))$, S. $\beta$-haemolyticus spp. $(19.0 \%$ ( $\mathrm{p}$ $<0.05))$, Actinomyces spp. $(8.8 \%(\mathrm{p}<0.05))$, Enterobacteriaceae $(26.3 \%(\mathrm{p}<0.05))$, Candida spp. $(26.3 \%(\mathrm{p}$ $<0.05)$ ). We observed a decrease in the Lactobacillus $s p$. colonization frequency in patients with periodontitis (by $18.6 \%(\mathrm{p}<0.05))$. 


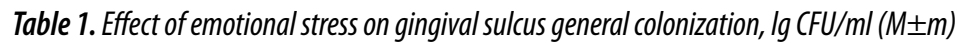

\begin{tabular}{|c|c|c|c|c|}
\hline \multirow[b]{2}{*}{ Indicators } & \multicolumn{2}{|l|}{ Intact gums } & \multicolumn{2}{|l|}{ Periodontitis } \\
\hline & Relative rest state & Voltage condition & Relative rest state & Voltage condition \\
\hline Aerobe colonization & $7,26 \pm 0,04$ & $7,40 \pm 0,03 \wedge$ & $7,79 \pm 0,03^{*}$ & $7,85 \pm 0,03^{*}$ \\
\hline Anaerobic colonization & $6,81 \pm 0,04$ & $7,07 \pm 0,08 \wedge$ & $7,73 \pm 0,03^{*}$ & $7,76 \pm 0,03^{*}$ \\
\hline
\end{tabular}

Notes: * - the reliability of indices differences in persons with periodontitis compared with a group of persons with intact gums, Student's $t$-test, $p<0.05$; $\wedge$ — the reliability of indices differences in persons under emotional stress compared with those in the state of relative rest, Student's $t$-test, $p<0.05$.

Table 2. Effect of emotional stress on gingival sulcus microbiota in people with no periodontal issues, \% of persons $/ \mathrm{lg}$ CFU/mI (M $\pm m)$

\begin{tabular}{l|l|l}
\hline Microorganisms & Relative rest state & Emotional stress \\
\hline S.viridans spp. & $95,5 / 6,91 \pm 0,09$ & $81,8 / 6,79 \pm 0,05$ \\
S.y-haemolyticus spp. & $81,8 / 6,81 \pm 0,10$ & $95,5 / 7,08 \pm 0,06$ \\
S.ß-haemolyticus spp. & $9,1 / 5,80 \pm 0,30$ & $9,1 / 6,51 \pm 0,39$ \\
Neisseria spp. & $36,4 / 5,88 \pm 0,26$ & $36,4 / 7,07 \pm 0,10 \wedge$ \\
Corynebacterium spp. & $31,8 / 5,84 \pm 0,25$ & $4,5 \wedge / 5,30$ \\
Lactobacillus spp. & $27,3 / 5,30 \pm 0,10$ & $9,1 \wedge / 5,25 \pm 0,25$ \\
S.epidermidis & $31,8 / 4,99 \pm 0,13$ & $31,8 / 5,65 \pm 0,23 \wedge$ \\
Bacillus spp. & $18,2 / 5,28 \pm 0,19$ & $9,1 / 5,50 \pm 0,20$ \\
Actinomyces spp. & $0 / 0$ & $0 / 0$ \\
S.aureus & $0 / 0$ & $0 / 0$ \\
Enterobacteriaceae & $0 / 0$ & $0 / 0$ \\
Candida spp. & $0 / 0$ & $0 / 0$ \\
\hline
\end{tabular}

Note: $\wedge$ — probability of frequency difference by the $\varphi$ Fisher criterion and lg $\mathrm{CFU} / \mathrm{ml}$, Student's $\mathrm{t}$-test, under emotional stress compared with those in the state of relative rest, $p<0.05$.

Here below we will look at how the gingival sulcus microbial status changed in eubiosis and dysbiosis in those exposed to emotional stress. In Group 1 (relative rest, the eubiotic state of the microbiota was identified) Lactobacillus spp. were detected 18.2\% less often $(\mathrm{p}<0.05)$, Corynebacterium spp. $-27.3 \%$ less often $(\mathrm{p}<0.05)$. At the same time, an increase in the microbial count of $S$. epidermidis $(4.6$ times $(\mathrm{p}<0.05)$ ) and Neisseria spp. (15.4 times $(\mathrm{p}<0.05))$ was detected.

In Group 2 we identified dysbiotic microbial status, where S. viridans spp. were detected $19.3 \%$ (p $<0.05)$ less often, Corynebacterium spp. $-17.5 \%(\mathrm{p}$ $<0.05)$ less often, S.epidermidis $(19.3 \%(\mathrm{p}<0.05))$ less often; on the other hand, Neisseria spp. and Enterobacteriaceae were found more often $(15.8 \%(\mathrm{p}<0.05)$ and $15.8 \%$ ( $\mathrm{p}<0.05)$, respectively), whereas the Neisseria spp. and Bacillus spp. colonization density was higher (1.9 times $(\mathrm{p}<0.05)$ and 6.6 times $(\mathrm{p}<0.05)$, respectively).

Describing the changes in the dentogingival gap microbiocenosis in case of emotional stress, note is to be made that 2 out of 22 persons in Group 1 (9.1\%) were found to have microbial populations imbalance, which could be described as a dysbiotic shift. All persons in Group 2 exposed to stress factors against the al- ready existing microbial dysbiotic changes, showed an increase in the microbial associations' imbalance. Out of the 45 persons examined, a dysbiotic shift was found in 18 people (31.6\%), another 27 showing Degree 1-2 dysbiosis (68.4\%).

Under emotional stress in individuals with eubiosis, the total gingival sulcus microbial colonization by aerobic and anaerobic microorganisms went up, with Lactobacillus spp. and Corynebacterium spp. found less often, while a higher microbial count was observed for S.epidermidis and Neisseria spp.

Individuals with dysbiosis showed no significant change in the total microbial load of the gingival sulcus, which remained at a high level; the Neisseria spp. and Bacillus spp. colonization density increased, while a decrease was observed in the detection rate for $S$. viridans spp. and Corynebacterium spp.; the Bacillus spp. and Enterobacteriacea colonization frequency increased as well.

Under emotional stress, $9.1 \%$ of persons with eubiosis revealed a dysbiotic shift in microbial populations. Those who already had microbial dysbiosis, demonstrated an increase in the microbial associations imbalance. Stress-induced alterations in the microbial status may be of different nature depending on the ini- 
tial state of the bacterial populations ratio in the biofilm. The eubiotic type of the microbial populations interaction is a stabilizing factor and offers adaptive maintenance of the biofilm homeostasis. The stressor effect along with an imbalance of the dentogingival gap microbial homeostasis leads to the aggravated imbalance in the microbial associations, which reveals itself as a decrease in the commensal microflora and an increase in the potentially pathogenic one.

The above shows that emotional stress had an impact on the quantitative and qualitative features of the gingival sulcus biofilm in both groups; this effect, however, was more significant in Group 2. The biofilm, which already manifested a microbial populations imbalance, responded to the stress factor with a more intense disturbance in the relationships between the symbiotic and potentially pathogenic microflora. The frequency of symbiotic S.viridans spp. colonization in individuals with the gingival sulcus biofilm dysbiosis was $69.5 \%$ lower $(p<0.05)$ compared with persons with eubiosis. In Group 2 opportunistic microorganisms (Bacillus spp., Actinomyces spp., S.aureus, Enterobacteriaceae, Candida spp.) were detected in the gingival biofilm significantly more often. The highest detection rate in Group 2 was observed for Bacillus spp. (47.0\% $(\mathrm{p}<0.05)$ more often than in Group 1), S.aureus (in $26.3 \%$ of cases $(\mathrm{p}<0.05))$, Actinomyces spp. ( $8.8 \%$ of cases $(\mathrm{p}<0.05)$ ), Enterobacteriaceae ( $42.1 \%$ of cases $(\mathrm{p}<0.05))$, Candida spp. $(28.1 \%$ of cases $(\mathrm{p}<0.05))$.

\section{CONCLUSIONS}

1. The resident microflora of each biotope performs numerous functions related not only to maintaining its functioning, yet also to the homeostasis as a whole. We have shown that the microbial status is a highly sensitive indicator system, which responds with quantitative and qualitative changes when subjected to external and internal factors. While law enforcement officers perform their public duties, they undergo an emotional load that is many times above normal, often reaching the level of emotional stress. Subjective view on the significance of a specific task can turn it into a strong emotional stressor [21, 22].

2. The study has revealed that psycho-emotional stress affects the gingival sulcus microbial status, although the level of this effect depends on the initial microbial associations balance. In case of eubiosis, the gingival biofilm is an important component of the natural anti-infectious protection system for periodontal tissues, ensuring the stability in the microecological homeostasis and the colonization resistance of this biological niche. Under dysbiosis, the initial microbial populations imbalance increases the susceptibility to pathogenic invasion [23-26].

3. Emotional stress affecting law enforcement officers, as our data shows, leads to a lower level and frequency of the gingival biofilm colonization with a stabilizing microflora. A decrease in the antagonistic action of these bacteria contributes to the gingival sulcus colonization with opportunistic microorganisms, and increased manifestations of dysbiosis, which is most intense in persons with a microbial populations initial imbalance. An imbalance in the resident microflora with a shift towards opportunistic microflora sets ground for the development of infectious diseases in the oral cavity.

\section{REFERENCES}

1. Bondarenko V. Microflora of the person: norm and pathology. Science in Russia. M., №1 (157). 2007. (In Russ.).

2. Bykov I.M., GiLmiyarova F.N., Domenyuk D.A., DMITRIENKo S.V., IVANYUTA S.O., BUDAYCHIEV

G. M-A. Evaluation of cariogenic situation in children with type 1 diabetes mellitus given the mineralizing potential of saliva and enamel resistance. Kubanskij nauchnyj medicinskij vestnik. 2018; 25(4): 22-36. (In Russ., English abstract). DOI: 10.25207 / 1608-62282018-25-4-22-36.

3. Domenyuk D.A., Davydov B.N., Gilmiyarova F.N., IVChenKo L.G., Vedeshina E.G. Diagnostic and prognostic value of crystalline structures of the oral fluid in children with anomalies of occlusion. Children's dentistry and prevention. 2017; Vol XXI; 2(61): 9-16. (In Russ.).

4. DAVYdov B.N., Domenyuk D.A., BYKov I.M., IVCHENKo L.G., DMITRIENKo S.V. Modern possibilities of clinical, laboratory, X-ray studies in preclinical diagnosis and prediction of the risk of developing periodontal diseases in children with diabetes mellitus type one. Part I. Periodontology. 2018; Vol. XXIV; 3-24 (88): 4-11. (In Russ.). DOI: 10.25636/ PMP.1.2018.3.1.

5. DOMENYUK D.A., ZeLenSKY V.A., RZHEPAKOVSKY I.V., ANFINOgENOva O.I., PushKIN S.V. Application of laboratory and $\mathrm{x}$-ray gentral studies un early diagnostics of metabolic disturbances of bone tissue in children with autoimmune diabetes mellitus. Entomology and Applied Science Letters. 2018; 5(4): 1-12.

6. Davydov B.N., GilmiYarova F.N., Domenyuk D.A., IVCHENKO L.G. Optimization of diagnostics of type I diabetes in children according to the results of cytomorphological studies of buccal epithelium and processes of oxidative stress in the oral cavity. Children's dentistry and prevention. 2017; Vol. XVI; 3(62): 9-18. (In Russ.).

7. Bykova N.I., Basov A.A., Melkonyan K.I., Alekseenko E.A., Popov K.A., BYkov I.M. Noninvasive monitoring for local immune and antioxidant 
resistance in patients with ischemic heart disease and type 2 diabetes. Medical news of North Caucasus, 2016; 11 (2): 147-149.

8. Bykov I.M., LAdutko A.A., Esaulenko E.E., ERICHEV I.V. Biochemistry of the oral and gingival fluid. Krasnodar, 2008. (In Russ.).

9. Domenyuk D.A., Zelensky V.A., DMitrienko S.V., Anfinogenova O.I., Pushkin S.V. Peculiarities of phosphorine calcium exchange in the pathogenesis of dental caries in children with diabetes of the first type. Entomology and Applied Science Letters. 2018; 5(4): 49-64.

10. BYkov I.M., IVChenko L.G., DOMENYUK D.A., Kostyukova N.Y., STorozhuk A.P., ILIJEv D.M. Salivary the level of proinflammatory cytokines in children with autoimmune diabetes mellitus in different phases of compensation endocrinopathy. Kuban Scientific Medical Bulletin. 2017; 24(4): 39-48. (In Russ., English abstract). DOI: 10.25207/1608-62282017-24-4-39-48

11. Bykov, I.M., Izhnina, E.V., Kochurova, E.V., LAPINA, N.V. Radiation-associated changes in salivation of patients with cancer of maxillofacial region. Dentistry. 2018; 97(1): 67-70.

12. Domenyuk, D.A. Semiquantitative evaluation of caries microflora in patients with dental and alveolar abnormalities and different severity of morphofunctional disturbances / D.A. Domenyuk, E.G. Vedeshina, V.A. Zelensky, K.G. Karakov, M.P. Porfiriadis // Medical news of North Caucasus. 2015. - Vol. 10. - № 3. - P. 238-241. (In Russ.) DOI: 10.14300/ mnnc.2015.10055.

13. Zakharevich N.V., Danilenko V.N. Serine/threonine protein kinases of bacteria are potential targets for regulation of human microbiota composition. Vestnik RGMU. 2017;(2): 20-29.(In Russ.).

14. Makeeva I. M., Bulgakov V. S., Nikolskaia I. A. An influence of the psychoemotional condition on the course of periodontitis. Scientific magazine "A health and education in XXI century".2008;1:140-141.(In Russ.).

15. Mikhailov B .V., Serdiuk A. I., Fedoseev V. A. Psychotherapy in general medicine: Clinical tutorial. Kharkov:Prapor, 2002. (In Russ.).

16. Pimenova M. N., Grechushinina N. N., Azova L. G., Netrusova A. I. A guide to practical lessons in microbiology. A tutorial. M.: MSU-pub.,1995. (In Russ.).

17. Pryanichnikova J.H. Examination stress and its prevention. Problems and prospects of educational development : materials of the VI International scientific conference. Perm. Mercure. 2015. P.184-186. (In Russ.).

18. Sidorenko E.V. Methods of mathematical processing in psychology. SPb.: OOO" Speech", 2007. (In Russ.).
19. Usmanova I.N., Tuigunov M.M., Gerasimova L.P., Kabirova M.F., Gubaidullin A.G., Gerasimova A.A., Husnarizanovna R.F. Role of opportunistic pathogenal microflora of the oral cavity in the development of inflammatory periodontal diseases and oral mucosa (literatura review). Human. Sport. Medicine. 2015. №2.(In Russ.).

20. Tsygan V. N., Skalnyi A.B., Mokeeva E.G. Sport, immunity, nutrition.SPb.: ELBI-SPb, 2011. (In Russ.).

21. Tsepov L.M., Nikolaev A.I., Nesterova M.M., NaKoneChNy D.A. Problem of an etiology of inflammatory generalized diseases of the parodont. Modern stomatology - efficiency of prevention and treatment. Nanotechnologies in stomatology. Materials of the conference dedicated to the 65th anniversary of the TverGMA. Tver,2014:310-319. (In Russ.).

22. Chereda V.V., Petrushanko T.A., Mamontova T.V. An influence of seasonality on the state of immunomicrobiocinosis of the oral cavityin young people. Actual problems of the modern medicine. Bulletin of the Ukrainian Medical Dental Academy.2014. №4 (48). (In Russ.).

23. De Iuliis V., Ursi S., Di Tommaso L.M., Caruso M., Marino A., Ercole S., Caputi S., Sinjari B., Festa F., Macri M., Martinotti S., Vitullo G., Toniato E.J.Comparative molecular analysis of bacterial species associated with periodontal disease. Biol.Regul.Homeost. Agents. 2016; 30(4): 1209-1215.

24. Razzouk S. Regulatory element sand genetic variations in periodontal diseases. Arch Oral Biol. 2016; 72 : 106-115. DOI: 10.1016/j.archoralbio.2016.08.015.

25. Gavrilova O.A., Domenyuk D.A. Specific features of oral cavity microbiocenosis in children using non-removable orthodontic appliances. Archiv EuroMedica, 2018; 8(2): 91-92.

26. Gavrilova O.A., Domenyuk D.A. Microbiological verification for the use of thermoplastics in prosthetic treatment of dentition issues in children. Archiv EuroMedica, 2018; 8(2): 88-90. 